Geophysical and Astrophysical Fluid Dynamics

Vol. 00, No. 00, 00 Month 2019, 1-18

\title{
The interaction of a mode-1 internal solitary wave with a step and the generation of mode-2 waves
}

\author{
Zihua Liu*, Roger Grimshaw and Edward Johnson \\ Department of Mathematics, University College London, London, WC1E 6BT, UK. \\ (Received 00 Month 20xx; final version received 00 Month 20xx)
}

\begin{abstract}
In this study we examine the transformation of a mode- 1 internal solitary wave incident on a bottom step, and the consequent generation of mode- 2 internal solitary waves. A linear long wave theory of mode coupling in the vicinity of the step is used to estimate the mode- 1 and mode- 2 wave reflection and transmission coefficients, and hence the energy fluxes. Away from the step, the wave evolution of the transmitted and reflected waves is simulated by the Korteweg-de Vries equation. Specific calculations are made using a three-layer fluid model. Three different regimes based on the layer thicknesses are examined and discussed in detail, for either depression or elevation mode- 1 incident waves. The common features found are that the transmitted waves (mainly mode-1) are the dominant part; most of the incident energy is transmitted and only a small part is reflected. The amplitudes of the generated mode- 2 waves and the reflected mode- 1 waves increase, when either the upper or middle layer thickness increases. When the lower layer is thin enough, the amplitude of the transmitted mode- 2 wave can be larger than the mode- 1 waves and the reflected energy can increase considerably which we infer may be due to a blocking effect of the step on the lower layer. The evolution away from the step is either fission into several solitary waves, or the development of a rarefaction wave followed by an undular bore, depending on the relative signs of the wave amplitudes and the nonlinear coefficient in the Korteweg-de Vries equation.
\end{abstract}

Keywords: Internal solitary waves; three-layer fluid; linear long wave theory; Korteweg-de Vries equation; mode-2 wave generation

\section{Introduction}

Oceanic internal solitary waves are commonly observed in coastal seas and over the continental slope. In analytical modelling they can be decomposed into an infinite set of vertical modes, where the term "mode" refers to a function describing the vertical structure. Mode- 1 internal waves have the fastest phase speed and the simplest internal vertical structure, typically a single maximum located near the pycnocline. Mode- 2 waves are the next fastest,with a phase speed usually around one third that of mode-1, and have an internal structure with two extrema. There are two different forms of mode- 1 internal solitary waves, depression and elevation waves, with the isopycnals displaced downwards and upwards respectively. For mode2 waves, the corresponding wave shapes are called convex when the upper (lower) isopycnals is displaced upward (downward), and are called concave when the upper (lower) isopycnals are displaced downward (upward). Observed mode-1 waves are depression waves when the near-surface isopycnals are displaced downwards.

Mode-1 internal solitary waves are the most commonly observed and studied, but our concern here is with the possible generation of mode- 2 waves by the interaction of a mode 1 wave with variable topography. Recently we (?) adapted the linear long-wave theory of ? which describes mode coupling by topography to determine the mode- 2 waves generated by an internal

*Corresponding author. Email: zihua.liu.15@ucl.ac.uk 


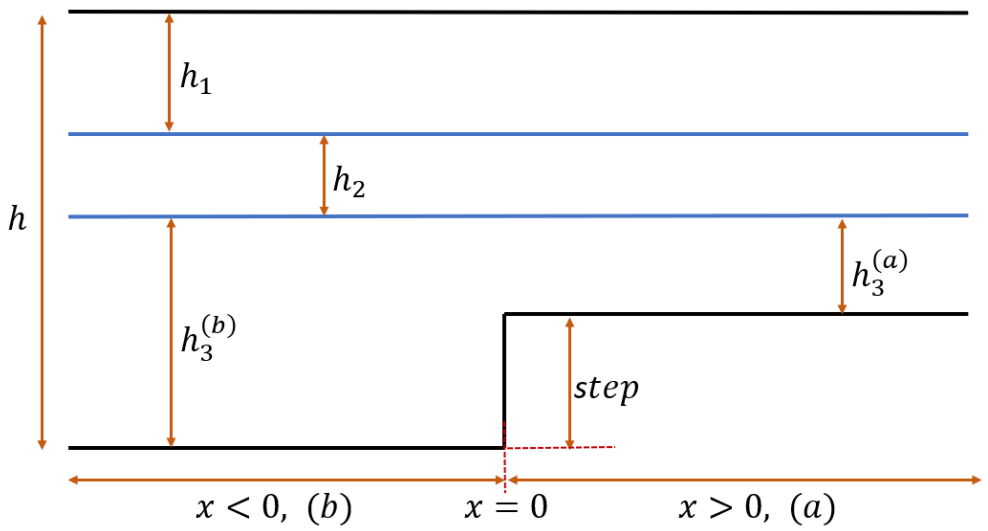

Figure 1. Sketch of the three-layer fluid system.

mode-1 solitary wave propagating over smooth slowly varying topography. However, the topography may not always be slowly varying, and so here we examine the alternative scenario when the topography is rapidly varying, modelled here by a bottom step. There have been several related studies on mode- 1 and mode- 2 solitary wave transformation by rapidly varying bottom topography. ? described an experimental and theoretical study of the interaction of a mode-1 internal solitary wave with a localised bottom topography (sill). ?? conducted laboratory experiments to investigate the evolution of mode- 1 incident internal solitary waves interaction with a steep slope varying from $30^{\circ}$ to $130^{\circ}$ in a stratified two-layer fluid system. ? studied the interaction of a mode- 1 internal wave with a bottom step in a two-layer flow in the framework of the Korteweg-de Vries and the Gardner equation (an extended version of the Korteweg-de Vries equation which includes both quadratic and cubic nonlinearity). ? developed Boussinesq-type equations for a two-layer flow with rapidly varying bottom topography. ??? considered the transformation of an internal solitary wave at a bottom step in the framework of a two-layer flow, based on numerical simulations of the fully nonlinear equations combined with a theoretical and numerical study of the Gardner equation. There are also studies on a mode- 2 solitary wave transformation over the rapidly varying bottom topography (see ? and ? for the interaction of a mode-2 solitary wave with a step and a narrow ridge respectively).

In this paper, we are concerned with the interaction of a mode- 1 internal solitary wave with a step and the generation of both transmitted and reflected mode- 1 and mode- 2 waves. In general an incident internal solitary wave will evolve into reflected and transmitted waves with many modes when it interacts with a step in the bottom topography. In the vicinity of the step, where the mode coupling occurs, we use linear long wave theory at a step with matching of the mass flux and pressure to determine the wave reflection and transmission coefficients, see sections 2 and 3 . Here we truncate this to two modes, mode- 1 and mode- 2 which is formally exact when the fluid stratification is an ideal three-layer fluid system (see figure 1), since then there are only mode- 1 and mode- 2 waves. This is implemented for various settings of thickness 
of each layer in the three-layer system (see table 1), and in each case we determine the wave amplitudes and the incident, reflected and transmitted energy fluxes. Then in section 4, we determine the wave evolution away from the step for both the reflected and transmitted mode1 and mode- 2 waves using the Korteweg-de Vries equation (1), similar to the strategy used by ? for a two-layer stratification. Our results are summarised in the discussion section 5, where we note in particular that a key parameter is the ratio of the mode- 2 to mode- 1 linear wave phase speed. A near-resonance configuration as this ratio increases towards unity, and this is the subject of an ongoing study.

\section{Korteweg-de Vries equation and linear long wave theory}

The solution structure is in two stages. Near the step, the time scales and space scales are short, so linear long wave theory can be used. Then the evolution towards and away from the step requires longer space and time scales, where nonlinearity will arise, and so we use the usual Korteweg-de Vries (KdV). In standard notation, for propagation over a constant depth $h$, this is

$$
A_{t}+c A_{x}+\frac{c Q_{x}}{2 Q} A+\mu A A_{x}+\delta A_{x x x}=0 .
$$

Here $\zeta=A(x, t) \phi(z ; h)$ is the leading order expression for the vertical particle displacement. The modal function $\phi(z ; h)$ is determined by, in the absence of a background shear flow, by the problem

$$
\begin{aligned}
& c^{2}\left(\rho_{0} \phi_{z}\right)_{z}+\rho_{0} N^{2} \phi=0 \quad \text { on } \quad-h<z<0 \text {, } \\
& \phi=0, \quad \text { at } \quad z=-h(x), \\
& c^{2} \phi_{z}=g \phi \quad \text { at } \quad z=0 .
\end{aligned}
$$

Here $N^{2}=-g \rho_{0 z} / \rho_{0}$, where $\rho_{0}(z)$ is the background density field. The modal equation $(2 a)$ solved subject $(2 b, c)$ determines the modal function $\phi(z ; h)$ and the linear long wave speed $c(h)$, where the $h$-dependence is parametric. The coefficients in (1) are given

$$
\mu=3 c^{2} \int_{-h}^{0} \rho_{0} \phi_{z}^{3} \mathrm{~d} z / I, \quad \delta=c^{2} \int_{-h}^{0} \rho_{0} \phi^{2} \mathrm{~d} z / I, \quad Q=c^{2} I,
$$

where

$$
I=2 c \int_{-h}^{0} \rho_{0} \phi_{z}^{2} \mathrm{~d} z .
$$

In general, the modal problem (2) determines an infinite set of modes, with real-valued phase speeds, that is $c^{2}>0$. Usually only the lowest mode- $1, \phi_{1}$ with the fastest linear phase speed $c_{1}$ is considered. Then the KdV equation (1) describes the evolution of the amplitude of this mode.

On the other hand, the linear long wave equations on a background density field $\rho_{0}(z)$ are

$$
\begin{aligned}
\rho_{0} u_{t}+p_{x} & =0, \\
p_{z}+\rho_{0} N^{2} \zeta & =0, \\
u_{x}+\zeta_{z t} & =0,
\end{aligned}
$$

which hold in the body of the fluid $-h<z<0$. The boundary conditions are

$$
\begin{aligned}
& \zeta=0 \quad \text { on } \quad z=-h, \\
& p=g \rho_{0} \zeta \quad \text { on } \quad z=0 \text {. }
\end{aligned}
$$


Here $\zeta, u, p$ are the vertical particle displacement, the horizontal velocity and the dynamic pressure respectively. The vertical velocity is $w=\zeta_{t}$ and the density perturbation is $\rho=$ $\rho_{0} N^{2} \zeta / g$. It is useful to define a flux variable

$$
q=\int_{-h}^{z} u \mathrm{~d} z
$$

and then (4c) implies that

$$
\zeta_{t}+q_{x}=0 .
$$

We are concerned here with a step topography, that is the depth $h$ is piecewise constant, $h^{(b)}, x<0$ and $h^{(a)}, x>0$, and we assume that $h^{(a)}<h^{(b)}$. The matching conditions across a step are continuity of pressure and mass flux, found by integrating (4a) and 7) across the step

$$
[p]_{x \uparrow 0}^{x \downarrow 0}=0, \quad[q]_{x \uparrow 0}^{x \downarrow 0}=0 .
$$

Both conditions hold in the domain $-h^{(a)}<z<0$ and the flux condition is supplemented by the requirement that $q(x \rightarrow 0-)=0,-h^{(b)}<z<-h^{(a)}$. Conservation of energy in this linear long wave limit is expressed by

$$
E_{t}+J_{x}=0
$$

in which

$$
E=\frac{1}{2}\left[\int_{-h}^{0}\left(\rho_{0} u^{2}+\rho_{0} N^{2} \zeta^{2}\right) \mathrm{d} z+\left.g \rho_{0} \zeta^{2}\right|_{z=0}\right], \quad J=\int_{-h}^{0} p u d z .
$$

Integrating across the step this yields

$$
[J]_{x \uparrow 0}^{x \downarrow 0}=0,
$$

expressing conservation of energy at the step.

\section{Mode generation at a step topography}

The theory of ? uses this linear long wave theory to decompose the wave field into a sum of vertical modes, noting that the full set of modal functions defined by (2) are complete. For our present purpose, we use a reduction of that theory restricted to just $N$ modes, so that the wave field is given by

$$
\begin{aligned}
\zeta & =\sum_{n=0}^{n=N} A_{n}(x, t) \phi_{n}(z ; h), & u & =\sum_{n=0}^{n=N} A_{n}(x, t) c_{n}(h) \phi_{n z}(z ; h), \\
q & =\sum_{n=0}^{n=N} A_{n}(x, t) c_{n}(h) \phi_{n}(z ; h), & p & =\rho_{0} \sum_{n=0}^{n=N} A_{n}(x, t) c_{n}^{2}(h) \phi_{n z}(z ; h) .
\end{aligned}
$$

Formally for completeness $N \rightarrow \infty$, but in the sequel we will truncate at either $N=1$ or $N=2$. Note that the modal functions and the phase speed are now indexed by the mode number, and have a parametric dependence on the depth $h$. Here mode- 0 is the surface wave mode, which in the Boussinesq and rigid lid approximation, is given by $\phi_{0}=(z+h) / h$ and $1 / c_{0}=0$. Although our main interest is in the generation of mode- 2 internal waves by a mode-1 internal wave, this surface mode needs to be included in layered fluid models. The 
modal functions satisfy the orthogonality conditions

$$
\int_{-h}^{0} \rho_{0} \phi_{m z} \phi_{n z} \mathrm{~d} z=\left\{\begin{array}{lll}
0, & \text { when } & m \neq n \\
I_{n}, & \text { when } & m=n
\end{array}\right.
$$

From ? when the depth is a constant, each mode satisfies a linear wave equation

$$
A_{n t t}-c_{n}^{2} A_{n x x}=0, \quad n=1,2 .
$$

Then for $x \neq 0$ the wave equations (13) have the solution

$$
A_{n}=\left\{\begin{array}{lll}
f\left(t-T_{n}^{b}\right) \delta_{k n}+R_{n} f\left(t+T_{n}^{b}\right) & \text { for } & x<0, \\
S_{n} f\left(t-T_{n}^{a}\right) & \text { for } & x>0
\end{array}\right.
$$

in which

$$
T_{n}^{(a),(b)}=x / c_{n}^{(a),(b)} .
$$

These describes an incident mode-k wave $(0 \leq k \leq N$, and reflected wave with a reflection coefficient $R_{n}$ and a transmitted wave with transmission coefficient $S_{n}$ for a set of mode- $n$ waves The total vertical displacements, velocity and pressure fields for $x \neq 0$ are given by (11). Our main concern is when $k=1$, an incident mode- 1 wave.

Next we apply the matching conditions (8) across the step. Using the modal expressions (11) we get that

$$
\begin{aligned}
& \sum_{n=0}^{n=N}\left(\delta_{k n}+R_{n}\right) c_{n}^{(b)^{2}} \phi_{n z}^{(b)}=\sum_{n=0}^{n=N} S_{n} c_{n}^{(a)^{2}} \phi_{n z}^{(a)}, \quad-h^{(a)}<z<0, \\
& \sum_{n=0}^{n=N}\left(\delta_{k n}-R_{n}\right) c_{n}^{(b)} \phi_{n}^{(b)}=\mathrm{H}\left(z+h^{(a)}\right) \sum_{n=0}^{n=N} S_{n} c_{n}^{(a)} \phi_{n}^{(a)}, \quad-h^{(b)}<z<0 .
\end{aligned}
$$

Here $\mathrm{H}(\bullet)$ is the Heaviside function, and we are assuming that $h^{(a)}<h^{(b)}$. Also, here $c_{n}^{(a),(b)}>0$ and so in the flux condition the sign of the reflected wave has been reversed. The pressure condition (15a) holds over the total depth just after the step, and the flux condition (15b) holds over the total depth just before the step. Clearly these conditions cannot be satisfied for all values of $z$, so instead they are applied in an averaged sense. That is in (15a) both sides are multiplied by $\rho_{0} \phi_{m z}^{(a)}$ and integrated over $-h^{(a)}<z<0$, for each $m=0, \cdots, N$. In (15b), we multiply both sides by $\rho_{0} N^{2} \phi_{m}^{(b)}=-c_{m}^{(b) 2}\left(\rho_{0} \phi_{m z}^{(b)}\right)_{z}$ and then integrate over $-h^{(b)}<$ $z<0$, again for each $m=0, \cdots, N$. Using the orthogonality conditions (12), (15a,b) yield respectively,

$$
\begin{aligned}
\sum_{n=0}^{n=N}\left(\delta_{k n}+R_{n}\right) c_{n}^{(b) 2} I_{m n}^{(b)} & =S_{m} c_{m}^{(a) 2} I_{m}^{(a)}, & & m=0, \cdots, N, \\
\left(\delta_{k m}-R_{m}\right) c_{m}^{(b)} I_{m}^{(b)} & =\sum_{n=0}^{n=N} S_{n} c_{n}^{(a)} I_{m n}^{(a)}, & & m=0, \cdots, N .
\end{aligned}
$$

Here the integrals are given by

$$
\begin{array}{llll}
I_{m}^{(a)}=\int_{-h^{(a)}}^{0} \rho_{0}{\phi_{m z}^{(a)}}^{2} \mathrm{~d} z, & I_{n}^{(a)}=\int_{-h^{(a)}}^{0} \rho_{0}{\phi_{n z}^{(a)}}^{2} \mathrm{~d} z, & I_{m n}^{(a)}=\int_{-h^{(a)}}^{0} \rho_{0} \phi_{m z}^{(b)} \phi_{n z}^{(a)} \mathrm{d} z \\
I_{m}^{(b)}=\int_{-h^{(b)}}^{0} \rho_{0}{\phi_{m z}^{(b)^{2}} \mathrm{~d} z,} I_{n}^{(b)}=\int_{-h^{(b)}}^{0} \rho_{0}{\phi_{n z}^{(b)^{2}} \mathrm{~d} z,}^{0} I_{m n}^{(b)}=\int_{-h^{(b)}}^{0} \rho_{0} \phi_{m z}^{(a)} \phi_{n z}^{(b)} \mathrm{d} z
\end{array}
$$


It is useful to note the following equality

$$
I_{m n}^{(a)}=I_{n m}^{(b)} .
$$

The $2 N+2$ equations (16a,b) form $2 N+2$ determine the $2 N+2$ unknowns $R_{n}, S_{n}$. From (10) the energy fluxes are the transmitted flux $J^{(a)}$ as $x \downarrow 0$, the incident flux $J^{(i n)}$ and the reflected flux $J^{(b)}$ as $x \uparrow 0$, each modulo a factor $f(t)^{2}$,

$$
J^{(a)}=\sum_{n=0}^{n=N} c_{n}^{(a) 3} I_{n}^{(a)} S_{n}^{2}, \quad J^{(i n)}=c_{k}^{(b) 3} I_{k}^{(b)}, \quad J^{(b)}=-\sum_{n=0}^{n=N} c_{n}^{(b) 3} I_{n}^{(b)} R_{n}^{2} .
$$

Note that each term in these sums has the form $c_{n} E_{n}$ where $E_{n}=c_{n}^{2} I_{n} A_{n}^{2}$ is the modal energy. A direct calculation from the system (16) shows that

$$
J^{(i n)}+J^{(b)}=J^{(a)},
$$

as expected from $(10)$.

If $h^{(a)}=h^{(b)}$ then $I_{m n}=\delta_{m n} I_{n}$ and the solution is just $S_{n}=\delta_{k n}, R_{n}=0$ as required. Before proceeding further, it is useful to note the reduction of the system (16) to just a single mode, the mode-k wave. This is achieved by omitting all terms in the sums in (11) and, (16a,b) except for $n=k$. The reduced system is easily solved to yield

$$
\begin{aligned}
R_{k}=\frac{\alpha_{k}-\beta_{k}}{\alpha_{k}+\beta_{k}}, & S_{k}=\frac{2}{\alpha_{k}+\beta_{k}}, & R_{k}^{2}+\sigma_{k} S_{k}^{2}=1, \\
\alpha_{k}=\frac{c_{k}^{(a) 2} I_{k}^{(a)}}{c_{k}^{(b) 2} I_{k k}^{(b)}}, & \beta_{k}=\frac{c_{k}^{(a)} I_{k k}^{(a)}}{c_{k}^{(b)} I_{k}^{(b)}}, & \sigma_{k}=\alpha_{k} \beta_{k}=\frac{c_{k}^{(a) 3} I_{k}^{(a)}}{c_{k}^{(b) 3} I_{k}^{(b)}} .
\end{aligned}
$$

Since $\sigma_{k}>0$, it follows that $R_{k}^{2}<1$ and $\sigma_{k} S_{k}^{2}<1$. Further, we have

$$
J^{(a)} / J^{(i n)}=\sigma_{k} S_{k}^{2}, \quad J^{(b)} / J^{(i n)}=-R_{k}^{2},
$$

and so

$$
J^{(i n)}-J^{(a)}=J^{(b)},
$$

as (19) requires. In particular, this limit can be used for a free surface wave, that is a mode-0 wave, with $k=0$, when the modal function $\phi_{0}(z) \approx(z+h) / h$ and speed $c_{0}=(g h)^{1 / 2}$. Then (20) becomes

$$
\alpha_{0}=1, \quad \beta_{0}=\sigma_{0}=c_{0}^{(a)} / c_{0}^{(b)}, \quad R_{0}=\frac{1-\beta_{0}}{1+\beta_{0}}, \quad S_{0}=\frac{2}{1+\beta_{0}},
$$

which agree with the well-known expressions for this case. However, this one mode truncation is not suitable for internal waves, even for a two-layer fluid as we shall now demonstrate.

For internal waves it is useful to invoke the commonly used Boussinesq approximation in which the density $\rho_{0}$ is constant except in the buoyancy term, that is $N^{2}$ retains a $z$ dependence. At the same time we also invoke the rigid lid approximation, so that (5b) is replaced by $\zeta=0$ at $z=0$ and in (2) the upper boundary condition becomes $\phi_{z=0}=0$. This filters out the surface wave mode, as in this limit, the modal function $\phi_{0} \approx(z+h) / h$ and the speed $c_{0} \approx(g h)^{1 / 2} \rightarrow \infty$. Nevertheless, a degenerate surface wave mode remains in the pressure field, with $\zeta_{0}=0, u_{0}=0$, but $p_{0}=P(t)$. Thus in the decomposition (11) the sum is over $n=1, \cdots N$, but in the pressure expression a term $P(t)$ replaces the $n=0$ term. The matching conditions $(15 \mathrm{a}, \mathrm{b})$ hold as before, but now for the sum $n=1, \cdots, N$ and in (14a) a constant term $P_{0}^{(a),(b)}$ is retained on the right and left hand sides respectively. The conditions 
(16a,b) hold as before, now truncated to the sum $n=1, \cdots, N$,

$$
\begin{aligned}
\sum_{n=1}^{n=N}\left(\delta_{k n}+R_{n}\right) c_{n}^{(b)}{ }^{2} I_{m n}^{(b)} & =S_{m} c_{m}^{(a)}{ }^{2} I_{m}^{(a)}, & & m=1, \cdots, N, \\
\left(\delta_{k m}-R_{m}\right) c_{m}^{(b)} I_{m}^{(b)} & =\sum_{n=1}^{n=N} S_{n} c_{n}^{(a)} I_{m n}^{(a)}, & & m=1, \cdots, N .
\end{aligned}
$$

From (15a) integration of both sides over $-h^{(a)}<z<0$ yields

$$
\left.\sum_{n=1}^{n=N}\left(\delta_{k n}+R_{n}\right) c_{n}^{(b)^{2}} \phi_{n}^{(b)}\right|_{z=-h^{(a)}}=h^{(a)}\left(P_{0}^{(b)}-P_{0}^{(a)}\right) .
$$

The restriction to a single mode-k wave again yields (20) for some $k=1, \cdots, N$. For instance for a two layer fluid with upper layer depth $h_{1}$ and lower layer depth $h_{2}, h=h_{1}+h_{2}$, a mode-1 wave has the modal function $\phi(z)=-z / h_{1}$ in the top layer and $\phi(z)=(z+h) / h_{2}$ in the bottom layer. The speed is given by $c^{2}=g^{\prime} h_{1} h_{2} / h$. Then we find that, with $k=1$,

$$
\alpha_{1}=1, \quad \beta_{1}=\sigma_{1}=c_{1}^{(a)} / c_{1}^{(b)}, \quad R_{1}=\frac{1-\beta_{1}}{1+\beta_{1}}, \quad \S_{1}=\frac{2}{1+\beta_{1}} .
$$

These agree with the analogous expressions in ?, and are identical with the corresponding expressions $(22 \mathrm{a}-\mathrm{d})$ for a surface wave. From $(23 \mathrm{a})$ we obtain

$$
S_{1} c_{1}^{(b)^{2}} \frac{\left(h_{2}^{(b)}-h_{2}^{(a)}\right)}{h_{2}^{(a)} h_{2}^{(b)}}=P_{0}^{(b)}-P_{0}^{(a)} .
$$

This expression agrees with (A.9) in Appendix A, found by a direct calculation for a two-layer fluid.

The system (23) is solved for a three-layer fluid in the Boussinesq approximation and a rigid upper boundary. The upper-layer depth is $h_{1}$, the middle-layer depth is $h_{2}$ and the lower layer depth is $h_{3}, h=h_{1}+h_{2}+h_{3}$. The same three-layer set-up exists in both $x<0$ and $x>0$ so that $h_{1}+h_{2}<h^{(a)}<h^{(b)}$. The modal structure is set out in Appendix B, and then the expressions (17) are readily evaluated. There are just two modes and a degenerate surface mode, which we do need to consider here. For the internal wave modes we find that, with $\rho_{0}=1$, for $N=2, m=1,2 ; n=1,2$,

$$
\begin{aligned}
I_{m n}^{(a)}=\frac{C_{1 m}^{(b)} C_{1 n}^{(a)}}{h_{1}}+\frac{D_{m}^{(b)} D_{n}^{(a)}}{h_{2}}+\frac{C_{2 m}^{(b)} C_{2 n}^{(a)}}{h_{3}^{(b)}}, & D_{m, n}^{(a, b)}=C_{1 m, 1 n}^{(a, b)}-C_{2 m, 2 n}^{(a, b)}, \\
I_{m n}^{(b)}=\frac{C_{1 m}^{(a)} C_{1 n}^{(b)}}{h_{1}}+\frac{D_{m}^{(a)} D_{n}^{(b)}}{h_{2}}+\frac{C_{2 m}^{(a)} C_{2 n}^{(b)}}{h_{3}^{(b)}}, & D_{m, n}^{(a, b)}=C_{1 m, 1 n}^{(a, b)}-C_{2 m, 2 n}^{(a, b)}, \\
I_{m, n}^{(a, b)}=\frac{C_{1 m, 1 n}^{(a, b)}}{h_{1}}+\frac{C_{2 m, 2 n}^{(a, b)}}{h_{3}^{(a, b)}}+\frac{D_{m, n}^{(a, b)}{ }^{2}}{h_{2}} . &
\end{aligned}
$$

The reflection and transmission coefficients for the internal wave modes in (14) are evaluated for a similar set up as in the companion paper (?). Figure 1 shows a diagram of this three-layer fluid system. We tested three cases with different layer thicknesses in the setup. In the first case, we set the total depth $h^{(b)}=500 \mathrm{~m}(x<0)$, the step height to $150 \mathrm{~m}$ and so $h^{(a)}=350 \mathrm{~m}$ $(x>0)$. With the upper layer depth $h_{1}=120 \mathrm{~m}$, we first allow the middle layer depth $h_{2}$ to vary from $40 \mathrm{~m}$ to $80 \mathrm{~m}$, see case 1 in table 1 . The outcome is shown in table 2 . With these values $h_{3}^{(a),(b)}>h_{1}$ in all cases, $H^{(a),(b)}<0(\mathrm{~B} .4)$, and so $C_{21}^{(a),(b)}=1,0<C_{11}^{(a),(b)}<1$ for 
Table 1. Setup of the three-layer fluid system for all three cases. (Total depth $h(\mathrm{~m})$, each layer's thickness $h_{1}, h_{2}, h_{3}^{(b)}$ and $h_{3}^{(a)}(\mathrm{m})$ and step thickness $(\mathrm{m})$.)

\begin{tabular}{c|cccccc}
\hline \hline Case & $h$ & $h_{1}$ & $h_{2}$ & $h_{3}^{(b)}$ & $h_{3}^{(a)}$ & step \\
\hline \hline \multirow{3}{*}{1} & 500 & 120 & 40 & 340 & 190 & 150 \\
& 500 & 120 & 60 & 320 & 170 & 150 \\
& 500 & 120 & 80 & 300 & 150 & 150 \\
\hline \hline \multirow{3}{*}{2} & 500 & 160 & 80 & 260 & 110 & 150 \\
& 500 & 180 & 80 & 240 & 90 & 150 \\
& 500 & 200 & 80 & 220 & 70 & 150 \\
\hline \hline \multirow{3}{*}{3} & 500 & 220 & 80 & 200 & 50 & 150 \\
& 500 & 240 & 80 & 180 & 30 & 150 \\
& 500 & 260 & 80 & 160 & 10 & 150 \\
\hline
\end{tabular}

Table 2. Case 1: reflection and transmission coefficients for (14) for an incident mode-1 wave, and the energy fluxes (18) in units of $\mathrm{ms}^{-3}$.

\begin{tabular}{c|ccccccc}
\hline \hline Case 1 & $S_{1}$ & $R_{1}$ & $S_{2}$ & $R_{2}$ & $J^{(i n)}$ & $J^{(a)}$ & $J^{(b)}$ \\
\hline \hline$h_{2}=40 \mathrm{~m}$ & 1.0290 & 0.0519 & -0.0462 & -0.0229 & 0.0244 & 0.0243 & -0.0001 \\
$h_{2}=60 \mathrm{~m}$ & 1.0221 & 0.0620 & -0.0706 & -0.0299 & 0.0233 & 0.0232 & -0.0001 \\
$h_{2}=80 \mathrm{~m}$ & 1.0100 & 0.0743 & -0.1017 & -0.0375 & 0.0224 & 0.0222 & -0.0002 \\
\hline
\end{tabular}

Table 3. Case 2: reflection and transmission coefficients for (14) for an incident mode-1 wave, and the energy fluxes (18) in units of $\mathrm{ms}^{-3}$.

\begin{tabular}{c|ccccccc}
\hline \hline Case 2 & $S_{1}$ & $R_{1}$ & $S_{2}$ & $R_{2}$ & $J^{(i n)}$ & $J^{(a)}$ & $J^{(b)}$ \\
\hline \hline$h_{1}=160 \mathrm{~m}$ & 1.1049 & 0.1087 & 0.1770 & -0.0603 & 0.0258 & 0.0255 & -0.0003 \\
$h_{1}=180 \mathrm{~m}$ & 1.1936 & 0.1328 & 0.2483 & -0.0772 & 0.0272 & 0.0267 & -0.0005 \\
$h_{1}=200 \mathrm{~m}$ & 1.2952 & 0.1646 & 0.3629 & -0.1007 & 0.0284 & 0.0276 & -0.0008 \\
\hline
\end{tabular}

mode- $1, C_{12}^{(a),(b)}=1,-1<C_{22}^{(a),(b)}<0$ for mode- 2 . The transmitted waves are the dominant part, and the reflected waves are quite small. In particular the reflected energy flux $J^{(b)}$ is less than $1 \%$ of the incident energy flux. The mode- 2 transmitted wave amplitude is quite small as expected, up to about $10 \%$ of the incident mode- 1 wave amplitude, and increases as the middle layer depth $h_{2}$ increases. The mode- 2 transmitted wave has the opposite polarity to the incident mode- 1 wave. Thus, if as is usual, the incident mode- 1 wave is a depression wave, the reflected and transmitted mode- 2 waves are waves of elevation, and hence are convex waves in the present set-up. The corresponding amplitudes determined from the mode- 1 approximation (25) when the mode- 2 wave is ignored are $R_{1}=0.0468,0.0539,0.0627, S_{1}=1+R_{1}$ as $h_{2}$ varies from $40 \mathrm{~m}$ to $80 \mathrm{~m}$ respectively. The agreement is quite good, and increases as $h_{2}$ decreases, as expected. But note that here $S_{1}$ decreases as $h_{2}$ decreases, whereas in the full result in table 2 we see that $S_{1}$ increases slightly as $h_{2}$ decreases. This can be attributed to the increases in the mode- 2 amplitudes as $h_{2}$ increases.

For the second case we fix the middle layer depth to be $h_{2}=80 \mathrm{~m}$ and then vary the upper layer depth in the range $h_{1}=160 \mathrm{~m}$ to $200 \mathrm{~m}$, see case 2 in table 1 . The outcome is shown in table 3 . With these values $h_{3}^{(b)}>h_{1}$ but $h_{3}^{(a)}<h_{1}$ in all cases, and so $H^{(b)}<0$ but $H^{(a)}>0$ (B.4). Thus $C_{21}^{(b)}=1,0<C_{11}^{(b)}<1$ as in the first case, but $C_{11}^{(a)}=1,0<C_{21}^{(a)}<1$ for mode-1. Similarly $C_{12}^{(b)}=1,-1<C_{22}^{(b)}<0$ as in the first case, but $C_{22}^{(a)}=1,-1<C_{12}^{(a)}<0$ for 
Table 4. Case 3: reflection and transmission coefficients for (14) for an incident mode-1 wave, and the energy fluxes (18) in units of $\mathrm{ms}^{-3}$.

\begin{tabular}{c|ccccccc}
\hline \hline Case 3 & $S_{1}$ & $R_{1}$ & $S_{2}$ & $R_{2}$ & $J^{(i n)}$ & $J^{(a)}$ & $J^{(b)}$ \\
\hline \hline$h_{1}=220 \mathrm{~m}$ & 1.3831 & 0.2089 & 0.5521 & 0.1360 & 0.0284 & 0.0269 & -0.0015 \\
$h_{1}=240 \mathrm{~m}$ & 1.4170 & 0.2770 & 0.8800 & 0.1961 & 0.0272 & 0.0247 & -0.0025 \\
$h_{1}=260 \mathrm{~m}$ & 1.2924 & 0.4105 & 1.4503 & 0.3341 & 0.0258 & 0.0203 & -0.0055 \\
\hline
\end{tabular}

mode-2. As in the previous case, the transmitted waves are still the dominant part, while the reflected waves are small. The absolute values of all the coefficients increase for fixed $h_{2}$ as $h_{1}$ increases. The reflected mode- 2 wave coefficient $R_{2}$ is still negative, but the transmitted mode- 2 wave coefficient $S_{2}$ is now positive, which means both the reflected and transmitted mode- 2 waves are convex, if the incident mode- 1 wave is a depression wave.

In the third case we again fix the middle layer depth to be $h_{2}=80 \mathrm{~m}$ but increase the the upper layer depth to vary in the range $h_{1}=220 \mathrm{~m}$ to $260 \mathrm{~m}$, see case 3 in table 1 . The outcome is shown in table 4 . With these values $h_{3}^{(a),(b)}<h_{1}$ in all cases, $H^{(a),(b)}>0($ B.4) and so $C_{11}^{(a),(b)}=1,0<C_{21}^{(a),(b)}<1$ for mode- $1, C_{22}^{(a),(b)}=1,-1<C_{12}^{(a),(b)}<0$ for mode- 2 . The transmitted waves are still the dominant part, but all the coefficients for both transmitted and reflected waves have increased. Note that the transmitted mode- 1 wave coefficient $S_{1}$ shows a slight decrease when $h_{1}$ increases to $260 \mathrm{~m}$. All the coefficients here are positive, so if the mode-1 incident wave is elevation (depression), both the reflected and transmitted mode-2 waves are concave (convex).

In all these cases, when the total depth and the top layer thickness $h_{1}$ is fixed, the absolute values of $R_{1}, R_{2}$ and $S_{2}$ increase as the middle layer thickness $h_{2}$ increases, while $S_{1}$ decreases. When $h_{2}$ is fixed, all amplitudes increase as $h_{1}$ increases, until the final value $\left(h_{1}=260 \mathrm{~m}\right)$, where $S_{1}$ shows a slight decrease. The other coefficients increase, and almost doubled when $h_{1}=240 \mathrm{~m}$. The mode- 2 transmitted wave amplitude is quite small initially, but increases when either layer $\left(h_{1}\right.$ or $\left.h_{2}\right)$ thickness becomes larger, and can become greater than the mode-1 incident wave amplitude. In the first and second cases, the incident energy flux $J^{(i n)}$ is mainly transmitted (over 95\%) with only a small part reflected, while the energy flux for each part increases when either $h_{2}$ or $h_{1}$ increases. But in the third case, the trend is opposite, the total incident energy flux $J^{(i n)}$ and transmitted energy flux $J^{(a)}$ decrease as $h_{1}$ increases, and the reflected energy flux $J^{(b)}$ increases. Over $20 \%$ total energy is reflected when $h_{1}=260 \mathrm{~m}$. This could be attributed to a blocking effect of the step on the lower layer.

\section{Evolution away from the step}

Once the reflected and transmitted amplitudes $R_{1,2}, S_{1,2}$ are determined, we can use the KdV equation (1) to describe the subsequent evolution, similarly to the strategy employed by ?. In both $x>0$ and $x<0$ the coefficients are constants and in particular $Q_{x}=0$. Then in $x>0$, for each mode $n=1,2$ we set

$$
\xi=T_{n}^{a}-t, \quad \tau=T_{n}^{(a)},
$$

and then the KdV equation (1) transforms to the asymptotically equivalent spatial form

$$
A_{\tau}+\nu A A_{\xi}+\lambda A_{\xi \xi \xi}=0,
$$

in which

$$
\nu=\mu_{n}^{(a)} / c_{n}^{(a)}, \quad \lambda=\delta_{n}^{(a)} / c_{n}^{(a)^{3}} .
$$


Table 5. KdV coefficients for case 1 for the transmitted $(x>0)$ and reflected $(x<0)$ waves

\begin{tabular}{cl|cccccc}
\hline \hline Case 1 & & $c_{1}$ & $\nu_{1}$ & $\lambda_{1}$ & $c_{2}$ & $\nu_{2}$ & $\lambda_{2}$ \\
\hline \hline$h_{2}=40 m$, & $x>0$ & 1.2171 & -0.0036 & 3619 & 0.4194 & 0.0627 & 2882 \\
& $x<0$ & 1.3493 & -0.0064 & 5028 & 0.4234 & 0.0625 & 3972 \\
\hline$h_{2}=60 m$, & $x>0$ & 1.1899 & -0.0024 & 3646 & 0.4970 & 0.0377 & 2888 \\
& $x<0$ & 1.3451 & -0.0054 & 5418 & 0.5047 & 0.0375 & 3915 \\
\hline$h_{2}=80 m$, & $x>0$ & 1.1568 & -0.0013 & 4265 & 0.5545 & 0.0253 & 2902 \\
& $x<0$ & 1.3380 & -0.0045 & 5781 & 0.5672 & 0.0250 & 3885 \\
\hline
\end{tabular}

The initial condition as $\tau \downarrow 0$ is found from the linear long wave solution of section 2 ,

$$
A(\tau \downarrow 0, \xi)=S_{n} f(-\xi), \quad n=1,2 .
$$

Similarly, in $x<0$, we set for each mode $n=1,2$,

$$
\xi=-T_{n}^{b}-t, \quad \tau=-T_{n}^{(b)},
$$

and then the KdV equation (1) transforms to the asymptotically equivalent spatial form

$$
A_{\tau}+\nu A A_{\xi}+\lambda A_{\xi \xi \xi}=0,
$$

in which

$$
\nu=\mu_{n}^{(b)} / c_{n}^{(b)}, \quad \lambda=\delta_{n}^{(b)} / c_{n}^{(b) 3} .
$$

The initial condition is now

$$
A(\tau \downarrow 0, \xi)=R_{n} f(-\xi), \quad n=1,2 .
$$

Note that although (29a) and (32a) appear to be identical, this is not the case as the variables $\tau, \xi$ and the coefficients $\nu, \lambda$ are indexed with $(a),(b), n$ (see $(29 \mathrm{~b}, \mathrm{c})$ and $(32 \mathrm{~b}, \mathrm{c}))$ which have been omitted for simplicity. The incident wave satisfies the same KdV equation (32) with $n=1$, but with $\xi=T_{n}^{b}-t, \tau=T_{n}^{(b)}$. We assume that this incident wave is a solitary wave, so that

$$
f(-\xi)=a \operatorname{sech}^{2}(K \xi), \quad \nu_{1}^{(b)} a=12 \lambda_{1}^{(b)} K^{2} .
$$

The simulations using (29) and (32) were carried out for the same parameter settings used to determine the reflection and transmission coefficients shown in tables 2, 3 and 4 . The corresponding $\mathrm{KdV}$ coefficients are shown in tables 5, 6 and 7, respectively. The incident wave amplitude is $a=-10$ or $10 \mathrm{~m}$. The respective outcomes are shown in figures 2,3 , 4 and 5 respectively. Here $\lambda>0$ in all cases, and so we expect fission when $a R_{1,2} \nu_{1,2}>$ $0, a S_{1,2} \nu_{1,2}>0$, or evolution into a rarefaction wave with a following undular bore when $a R_{1,2} \nu_{1,2}<0, a S_{1,2} \nu_{1,2}<0$. This is indeed what occurs. In the case of fission, although formulas are available to determine the number and amplitudes of the emitted solitary waves (see ??? and the review by ? for instance) their use here is rather too complicated in view of the large first casse of $h_{1}$ stem $h_{3}$ throuroughout, we set the initial mode-1 incident wave to be a depression wave, with amplitude $a=-10 \mathrm{~m}$, so $a R_{1,2} \nu_{1,2}^{(b)}>0$ and $a S_{1,2} \nu_{1,2}^{(a)}>0$ (see table 2 and 5 ). There are no polarity changes, and so fission occurs for both the reflected waves and the transmitted waves, see figure 2 . In the second case, $h_{1}<h_{3}(x<0)$ but $h_{1}>h_{3}(x>0)$, while the initial wave amplitude is again $a=-10 \mathrm{~m}$, a depression wave. From table 3 and $6, a R_{1,2} \nu_{1,2}^{(b)}>0$ and $a S_{2} \nu_{2}^{(a)}>0$, again indicating wave fission for the reflected mode- 1 and mode- 2 waves, and for the transmitted mode- 2 wave. But $a S_{1} \nu_{1}^{(a)}<0$, so the transmitted mode- 1 wave evolves into a rarefaction wave followed by an undular bore, as shown in figure 3 . The third case is much more complicated. The initial incident mode- 1 wave is now an 

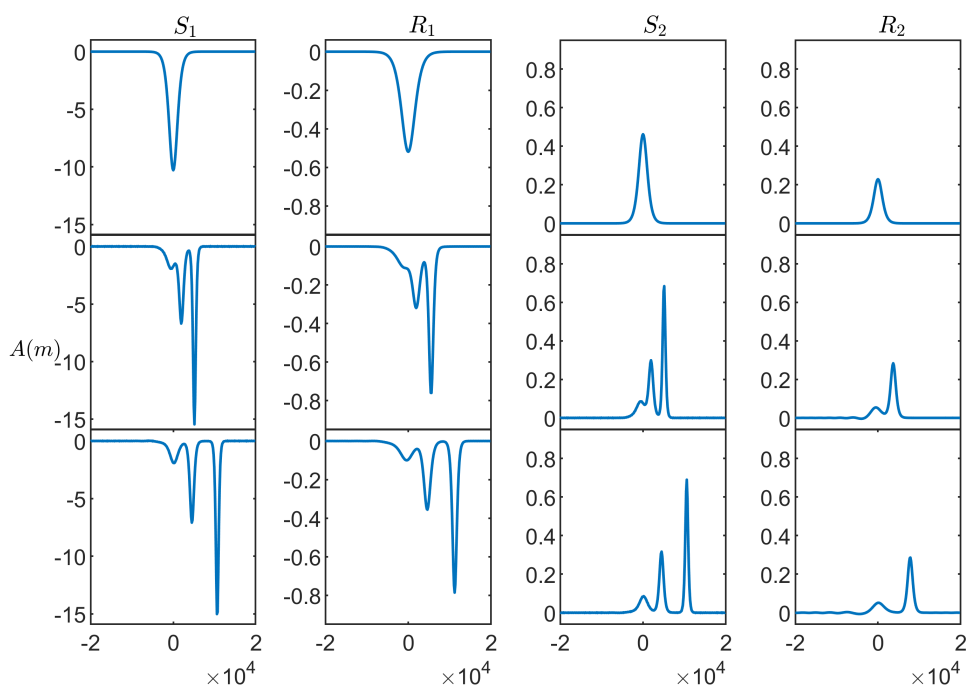

Figure 2. Case 1: Wave evolution for an incident mode-1 wave interaction with a step in an ideal three-layer fluid system, with a middle layer thickness $h_{2}=40 \mathrm{~m}$. The panels labelled $S_{1}, R_{1}, S_{2}, R_{2}$ are for the transmitted mode- 1 wave, the reflected mode- 1 wave and the transmitted mode- 2 wave, the reflected mode- 2 wave respectively.

Table 6. KdV coefficients for case 2 for the transmitted $(x>0)$ and reflected $(x<0)$ waves.

\begin{tabular}{cl|cccccc}
\hline \hline Case 2 & & $c_{1}$ & $\nu_{1}$ & $\lambda_{1}$ & $c_{2}$ & $\nu_{2}$ & $\lambda_{2}$ \\
\hline \hline$h_{1}=160 m$, & $x>0$ & 1.1478 & 0.0022 & 4293 & 0.5526 & -0.0242 & 2874 \\
& $x<0$ & 1.4156 & -0.0024 & 5577 & 0.5763 & 0.0283 & 4090 \\
\hline$h_{1}=180 m$, & $x>0$ & 1.1161 & 0.0039 & 4397 & 0.5452 & -0.0210 & 2770 \\
& $x<0$ & 1.4371 & -0.0014 & 5527 & 0.5785 & 0.0293 & 4140 \\
\hline$h_{1}=200 m$, & $x>0$ & 1.0655 & 0.0055 & 4586 & 0.5309 & -0.0156 & 2581 \\
& $x<0$ & 1.4478 & -0.0005 & 5503 & 0.5795 & 0.0301 & 4164 \\
\hline
\end{tabular}
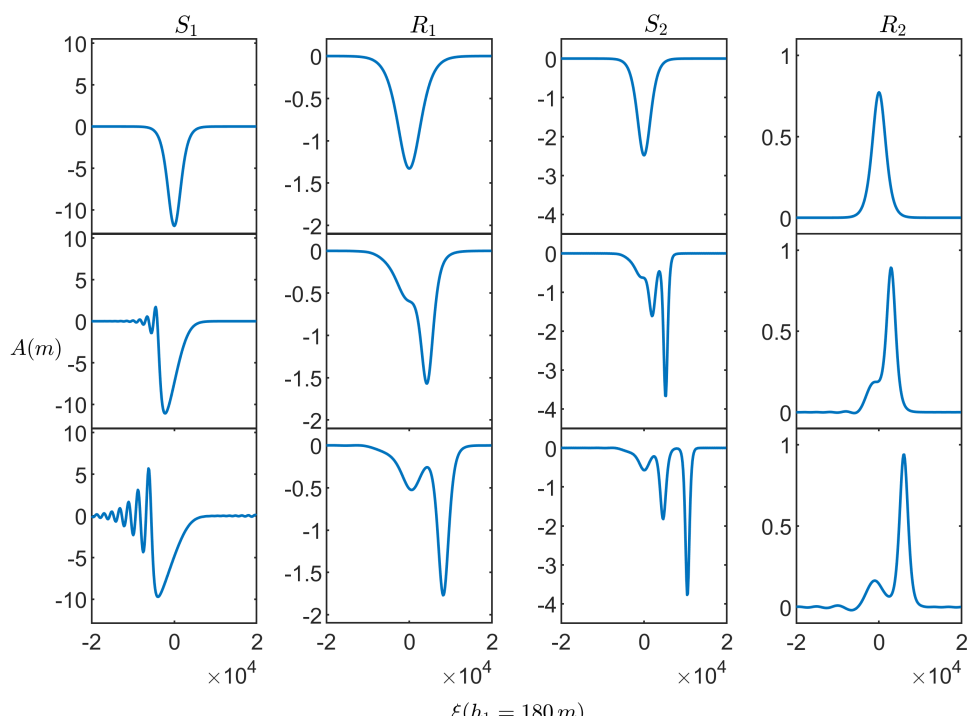

Figure 3. Case 2: Wave evolution for an incident mode-1 wave interaction with a step in an ideal three-layer fluid system, with a top layer thickness $h_{1}=180 \mathrm{~m}$. The panels labelled $S_{1}, R_{1}, S_{2}, R_{2}$ are for the transmitted mode- 1 wave, the reflected mode- 1 wave and the transmitted mode- 2 wave, the reflected mode- 2 wave respectively. 
Table 7. KdV coefficients for case 3 for the transmitted $(x>0)$ and reflected $(x<0)$ waves.

\begin{tabular}{cl|cccccc}
\hline \hline Case 3 & & $c_{1}$ & $\nu_{1}$ & $\lambda_{1}$ & $c_{2}$ & $\nu_{2}$ & $\lambda_{2}$ \\
\hline \hline$h_{1}=220 m$, & $x>0$ & 0.9962 & 0.0071 & 4895 & 0.5034 & -0.0054 & 2271 \\
& $x<0$ & 1.4478 & 0.0005 & 5503 & 0.5795 & -0.0301 & 4164 \\
\hline$h_{1}=240 m$, & $x>0$ & 0.9113 & 0.0085 & 5346 & 0.4452 & 0.0186 & 1821 \\
& $x<0$ & 1.4371 & 0.0014 & 5527 & 0.5785 & -0.0293 & 4140 \\
\hline$h_{1}=260 m$, & $x>0$ & 0.8235 & 0.0110 & 5767 & 0.2960 & 0.1265 & 1399 \\
& $x<0$ & 1.4156 & 0.0024 & 5577 & 0.5763 & -0.0283 & 4090 \\
\hline
\end{tabular}
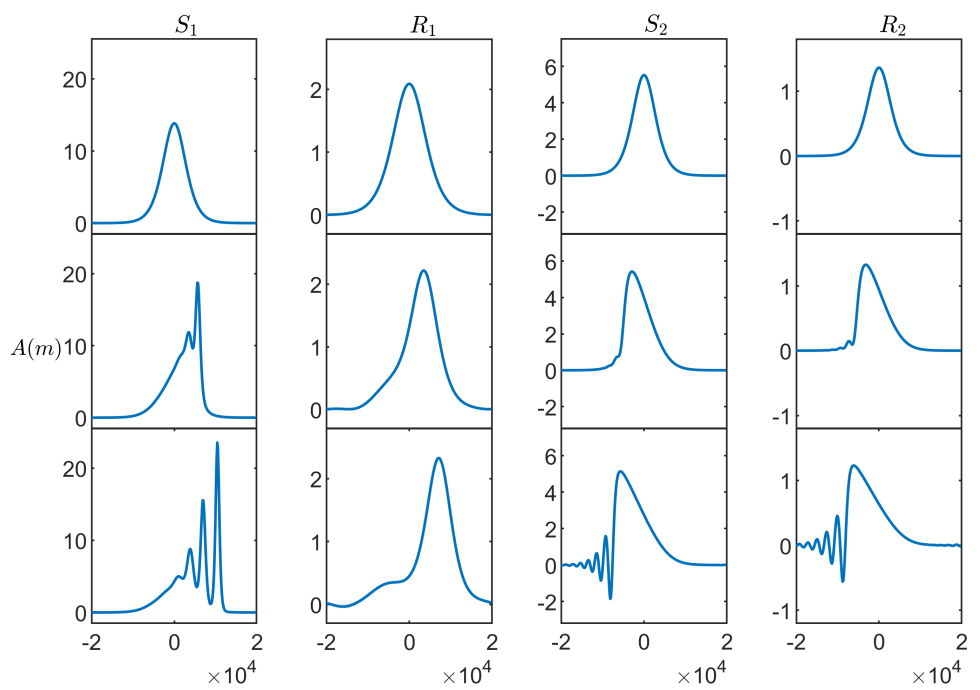

$\xi\left(h_{1}=220 \mathrm{~m}\right)$

Figure 4. Case 3: Wave evolution for an incident mode-1 wave interaction with a step in an ideal three-layer fluid system, with a top layer thickness $h_{1}=220 \mathrm{~m}$. The panels labelled $S_{1}, R_{1}, S_{2}, R_{2}$ are for the transmitted mode-1 wave, the reflected mode- 1 wave and the transmitted mode- 2 wave, the reflected mode- 2 wave respectively.
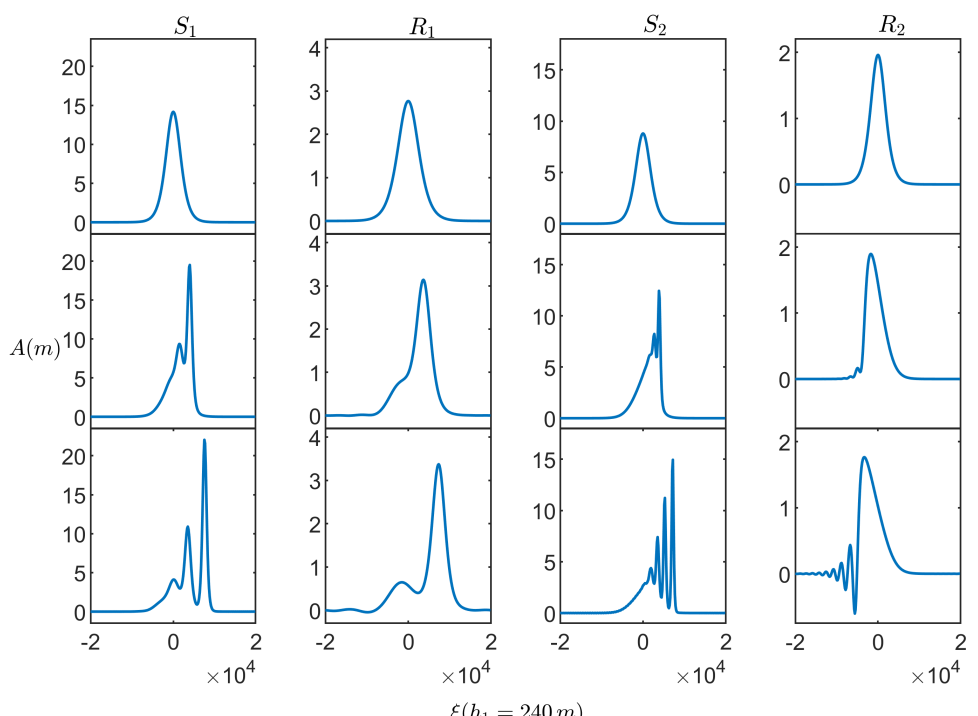

Figure 5. Case 3: Wave evolution for an incident mode-1 wave interaction with a step in an ideal three-layer fluid system, with a top layer thickness $h_{1}=240 \mathrm{~m}$. The panels labelled $S_{1}, R_{1}, S_{2}, R_{2}$ are for the transmitted mode- 1 wave, the reflected mode- 1 wave and the transmitted mode- 2 wave, the reflected mode- 2 wave respectively. 
elevation wave with amplitude $a=10 \mathrm{~m}$, because $h_{1}>h_{3}$ in the whole region. From tables 4 and 7 , when $h_{1}=220 \mathrm{~m}, a R_{1} \nu_{1}^{(b)}>0, a S_{1} \nu_{1}^{(a)}>0$, but $a R_{2} \nu_{2}^{(b)}<0, a S_{2} \nu_{2}^{(a)}<0$, so wave fission only occurs for mode- 1 waves, while the mode- 2 waves transform from an initial elevation wave (concave wave with respect to the mode- 2 wave shape) to a rarefaction wave with a following undular bore, see figure 4 . However, as $h_{1}$ increases further $\left(h_{1}=240,260 \mathrm{~m}\right)$, $a R_{1} \nu_{1}^{(b)}>0, a S_{1,2} \nu_{1,2}^{(a)}>0$, and only $a R_{2} \nu_{2}^{(b)}<0$. As shown in figure 5 , the reflected mode- 2 wave develops into a rarefaction wave, but the other waves form more solitary waves due to wave fission.

\section{Discussion}

In this paper, we have examined the impact of a weakly nonlinear mode- 1 internal solitary wave incident on a step in the framework of a three-layer fluid model, with layer depths $h_{1}, h_{2}, h_{3}^{(a),(b)}$, where we recall that the indices $(a),(b)$ refer to the regimes after and before the step. The linear long wave theory of section 2 and 3 with mode coupling in the vicinity of the step is used to estimate the mode- 1 and mode- 2 wave reflection $\left(R_{1,2}\right)$ and transmission $\left(S_{1,2}\right)$ coefficients, and hence the incident energy flux $J^{(i n)}$, reflected energy flux $J^{(b)}$ and transmitted energy flux $J^{(a)}$. In this linear theory, the matching conditions at the step are valid for an arbitrary step height but for sufficiently small waves. In practice, larger step heights will generate larger waves with significant vertical velocities, and the flow may separate at the step and generate vortical structures (see ??). There are no evanescent modes in this linear long wave theory. A full analysis even at the linear level, would require evanescent modes in order to satisfy the boundary conditions at the step. But these would decay exponentially away from the step, and do not carry any mass or energy. Hence in this long wave analysis they do not contribute to the matching conditions that we have used. Away from the step, the wave evolution is simulated by the KdV equation (1) and the results are described in section 4. In Appendix $\mathrm{C}$ we describe briefly the analogous results for an incident mode-2 wave.

Three different regimes of mode-1 incident internal solitary wave interaction with a step were identified and the results are summarised as follows:

Case 1: the upper layer depth $h_{1}=120 \mathrm{~m}$ is fixed, and $h_{1}<h_{3}^{(a, b)}$ throughout, while the middle layer thickness is very thin but increases for each trial.

The transmitted waves are the dominant part and the reflected waves are very small. The transmitted mode- 2 wave amplitude is also quite small as expected compared to the transmitted mode-1 wave, up to about $10 \%$. The reflected energy flux $\left(J^{(b)}\right)$ is less than $1 \%$ of the incident energy flux $\left(J^{(i n)}\right)$. As $h_{2}$ increases, the mode- 2 wave amplitude coefficients $\left(S_{2}\right.$, $\left.R_{2}\right)$ and the reflected mode- 1 wave amplitude coefficients $\left(R_{1}\right)$ increase, while the transmitted mode- 1 wave coefficients $\left(S_{1}\right)$ decrease. The energy flux trend is consistent with the wave amplitude trends, where the incident and transmitted wave energy flux decreases, and the reflected wave energy flux increases. Using the nonlinear coefficients in the KdV equation for both mode-1 waves $\left(\nu_{1}^{(a),(b)}\right)$ and mode- 2 waves $\left(\nu_{2}^{(a),(b)}\right)$, if the incident mode- 1 wave is depression (the initial wave amplitude $a=-10 \mathrm{~m}$ ), then $a R_{1,2} \nu_{1,2}^{(b)}>0$ and $a S_{1,2} \nu_{1,2}^{(a)}>0$, so wave fission occurs for both the reflected and transmitted waves.

Case 2: the middle layer depth $h_{2}=80 \mathrm{~m}$ is constant, and $h_{1}$ increases so that $h_{1}<h_{3}^{(b)}$ but $h_{1}>h_{3}^{(a)}$.

As in case 1 , the dominant part is the transmitted waves, but although the transmitted mode- 2 wave is still small, it can be up to $30 \%$ of the transmitted mode- 1 wave. All the wave amplitudes in this case are correspondingly larger than those in the first case, and the energy 
flux for each part is stronger than in the first case, but still only a small part (less than 5\%) is reflected. As $h_{1}$ increases, both the reflected and transmitted wave amplitudes increase, and the energy flux changes in the same way. In the region of the incident mode- 1 wave, $h_{1}<h_{3}^{(b)}$, so we assume again that the initial wave amplitude is a depression, $a=-10 \mathrm{~m}$, and then $a R_{1,2} \nu_{1,2}^{(b)}>0$ and $a S_{2} \nu_{2}^{(a)}>0$, but $a S_{1} \nu_{1}^{(a)}<0$. Hence, the reflected waves ( both mode- 1 and mode-2) and the transmitted mode- 2 waves fission to secondary solitons, but the transmitted mode-1 wave evolves into a rarefaction wave followed by an undular bore.

Case $3: h_{2}=80 \mathrm{~m}$ as in case 2 , and $h_{1}>h_{3}^{(a, b)}$ throughout.

As before, the transmitted waves are the main part, but now significantly the transmitted mode- 2 wave is not small but instead comparable or even exceeding the transmitted mode-1 wave amplitude. As in case $1, R_{1,2}$ and $S_{2}$ increase as $h_{1}$ increases, while $S_{1}$ increases slightly but then decreases. The energy flux here is similar to that in the first two cases, but now the reflected energy flux can be over $20 \%$, which is consistent with the increased reflected wave amplitudes. The wave evolution dynamics is much more complicated in this case. As $h_{1}>h_{3}^{(a),(b)}$, we set the incident mode- 1 wave to be an elevation with the initial wave amplitude $a=10 \mathrm{~m}$. When $h_{1}=220 \mathrm{~m}, a R_{1} \nu_{1}^{(b)}>0, a S_{1,2} \nu_{1,2}^{(a)}>0$, but $a R_{2} \nu_{2}^{(b)}<0, a S_{2} \nu_{2}^{(a)}<0$, so there is only elevation waves with fission for mode- 1 , but for mode- 2 the waves are concave, and evolve into a rarefaction with a following undular bore.

From this study, we see that usually the transmitted waves (mainly mode-1) are the dominant part, and so most of the incident energy is transmitted and only a small part is reflected. When the lower layer is thin enough, the reflected energy can increase a lot which we infer may be due to a blocking effect of the step on the lower layer. The amplitudes of the generated mode- 2 waves and the reflected mode- 1 waves increase, when either the upper or middle layer thickness increases. The wave dynamics of the mode- 1 waves depends on the upper and lower layer thicknesses, when the middle layer is thin. The wave dynamics of mode- 2 waves is more complicated, and involves in the distribution of each layer thickness. We have chosen here to use a three-layer fluid model to assess this interplay between mode- 1 and mode- 2 waves, but to extrapolate these results to more general stratifications (noting that our formulation can accommodate any specified stratification), it is useful to summarise the overall pattern using just the ratio of the mode- 1 and mode- 2 wave speeds,

$$
R_{\text {speed }}=\frac{c_{2}}{c_{1}}=\frac{\sqrt{K+1-\left(H^{2}+1\right)^{1 / 2}}}{\sqrt{K+1+\left(H^{2}+1\right)^{1 / 2}}},
$$

in which

$$
H=\frac{h_{2}}{2}\left(\frac{1}{h_{3}}-\frac{1}{h_{1}}\right), \quad K=\frac{h_{2}}{2}\left(\frac{1}{h_{3}}+\frac{1}{h_{1}}\right),
$$

and where we have used (B.3c) in appendix B to evaluate this for a three-layer fluid.

Figure 6 shows this ratio as a function of the two parameters $h_{2} / h_{1}, h_{2} / h_{3}$ for the three-layer fluid model. Note that $0<R_{\text {speed }}<1$ always and we expect significant generation of mode-2 waves when $R_{\text {speed }} \rightarrow 1$; that is when there is a near-resonance between a mode- 1 wave and a mode- 2 wave. As expected this possible resonance occurs as $h_{2} / h_{1}$ and $h_{2} / h_{3}$ increase with $h_{1} \approx h_{3}$. The enlarged view in figure 6 indicates $R_{\text {speed }}$ for the three cases we have examined, which are in the range from 0.3 and 0.5 , typical of observed mode- 2 and mode- 1 wave speeds. In general, from the specific results presented our three cases in section 3 , where $h_{2} / h_{1}$ and/or $h_{2} / h_{3}$ are varied, we see that there is an increase in mode- 2 amplitudes as $R_{\text {speed }}$ increases. 


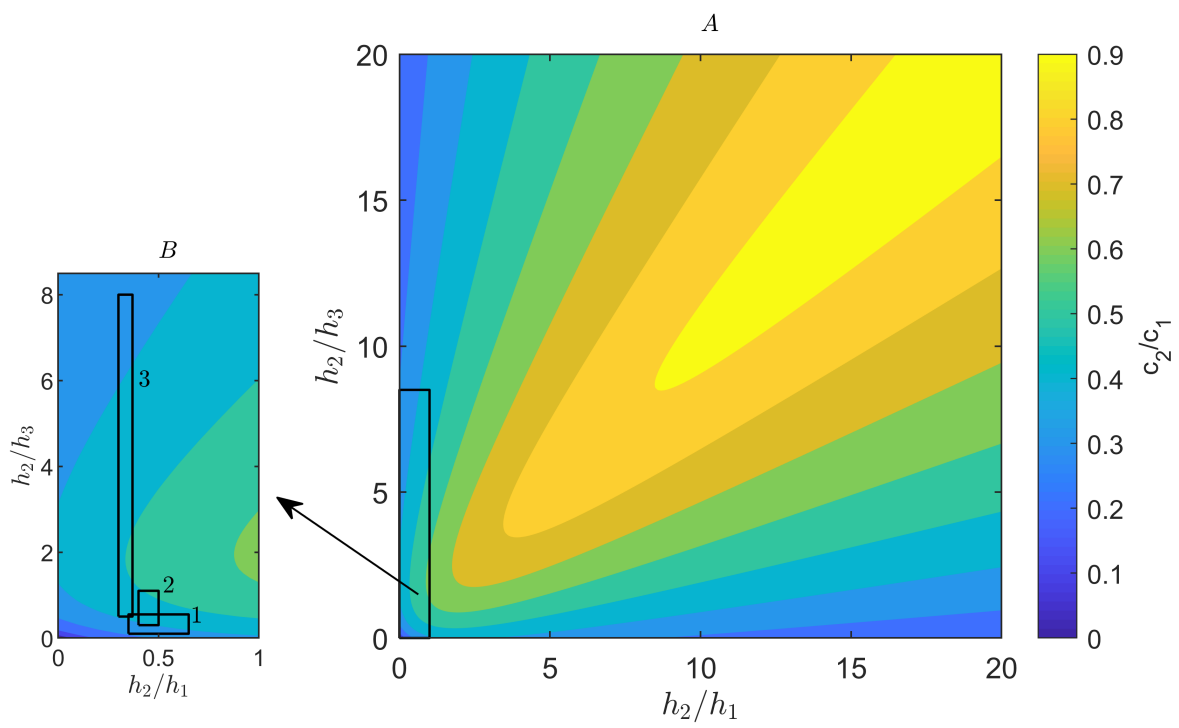

Figure 6. A: The ratio $R_{\text {speed }}$ (35a) of mode-2 and mode- 1 wave speeds for a three-layer fluid model as a function of $h_{2} / h_{1}$ and $h_{2} / h_{3}$. B: The enlarged view of the selected rectangle region in A. Rectangles 1,2 and 3 in B correspond to case 1 , case 2 and case 3 .

\section{Appendix A: Two-layer fluid system}

The linear long wave equations are, in the Boussinesq and rigid lid approximation,

$$
\begin{aligned}
u_{t}^{(1)}+\mathcal{P}_{x} & =0, \\
u_{t}^{(2)}+\mathcal{P}_{x}+g^{\prime} \zeta_{x} & =0, \\
-\zeta_{t}+\left(h_{1} u^{(1)}\right)_{x} & =0, \\
\zeta_{t}+\left(h_{2} u^{(2)}\right)_{x} & =0,
\end{aligned}
$$

and $h=h_{1}+h_{2}$. The total pressure is given by $p_{\text {total }}=p_{0}(z)+p$ :

$$
\begin{gathered}
p_{0}= \begin{cases}-g \rho_{1} z, & -h_{1}<z<0, \\
-g \rho_{2}\left(z+h_{1}\right)+g \rho_{1} h_{1}, & -h<z<-h_{1},\end{cases} \\
p=\left\{\begin{array}{lr}
\mathcal{P}, & -h_{1}<z<0, \\
\mathcal{P}+g^{\prime} \zeta, & -h<z<-h_{1} .
\end{array}\right.
\end{gathered}
$$

The mode- 1 modal function is

$$
\phi_{1}=\left\{\begin{array}{ll}
-z / h_{1}, & -h_{1}<z<0, \\
(z+h) / h_{2}, & -h<z<-h_{1},
\end{array} \quad c_{1}^{2}=\frac{g^{\prime} h_{1} h_{2}}{h} .\right.
$$

The corresponding pressure and velocity fields are

$$
\begin{aligned}
& p_{1}= \begin{cases}-\left(c_{1}^{2} / h_{1}\right) \zeta_{1}, & -h_{1}<z<0 \\
\left(c_{1}^{2} / h_{2}\right) \zeta_{1}, & -h<z<-h_{1},\end{cases} \\
& u_{1}^{(1)}=-\left(c_{1} / h_{1}\right) \zeta_{1}, \quad-h_{1}<z<0, \\
& u_{1}^{(2)}=\left(c_{1} / h_{2}\right) \zeta_{1}, \quad-h<z<-h_{1} \text {. }
\end{aligned}
$$


This suggests to put

$$
\begin{aligned}
\mathcal{P} & =P-\left(c_{1}^{2} / h_{1}\right) \zeta, \\
u_{t}^{(1)}+P_{x}-\left(c_{1}^{2} / h_{1}\right) \zeta_{x} & =0, \\
u_{t}^{(2)}+P_{x}+\left(c_{1}^{2} / h_{2}\right) \zeta_{x} & =0 .
\end{aligned}
$$

This $4 \times 4$ system has an internal mode speed $c_{1}$ and modal function $\phi_{1}$ and $P_{1}=0$, and a degenerate "surface" mode with speed $c_{0} \rightarrow \infty$ and a modal solution in which $\zeta_{0}=0, u^{(1,2)}=$ $0, P_{0}=P_{0}(t) \neq 0$. Across the step, the matching conditions are

$$
[\zeta]_{(b)}^{(a)}=0, \quad[\mathcal{P}]_{(b)}^{(a)}=0, \quad\left[h_{1} u_{1}\right]_{(b)}^{(a)}=0, \quad\left[h_{2} u_{2}\right]_{(b)}^{(a)}=0 .
$$

Implementation of these yields the expression for mode- 1 and continuity of $\mathcal{P}$ as in ?. But note that the degenerate surface mode $P_{0}$ is not continuous and instead is found from

$$
[P]_{(b)}^{(a)}=\zeta\left(\left[c_{1}^{2}\right]_{(b)}^{(a)} / h_{1}\right) .
$$

\section{Appendix B: Three-layer fluid system}

Consider a three-layer fluid model, as described by ? for a different purpose. We assume that the background density field is given by

$$
\begin{aligned}
\rho_{0}(z)= & \left(\rho_{2}+\Delta \rho\right) \mathcal{H}\left(-z-h_{1}-h_{2}\right) \\
& +\rho_{2} \mathcal{H}\left(-z-h_{1}\right) \mathcal{H}\left(z+h_{1}+h_{2}\right)+\left(\rho_{2}-\Delta \rho\right) \mathcal{H}\left(z+h_{1}\right),
\end{aligned}
$$

where $h_{1}, h_{2}$ and $h_{3}$ are the thickness of the three layers from top to bottom, respectively. $h=h_{1}+h_{2}+h_{3}, \rho_{2}$ is the density of the middle layer and $\Delta \rho$ is the density difference across each interface. $\mathcal{H}(\bullet)$ is the Heaviside function. We will also invoke the Boussinesq approximation and replace the upper boundary with a rigid lid. Since $N^{2}=0$ in each layer, from 2 the modal function is given by

$$
p=\left\{\begin{array}{lr}
-C_{1} z / h_{1}, & -h_{1} \leq z \leq 0, \\
C_{1}\left(z+h_{1}+h_{2}\right) / h_{2}-C_{2}\left(z+h_{1}\right) / h_{2}, & -h_{1}-h_{2}<z<-h_{1}, \\
C_{2}(z+h) / h_{3}, & -h \leq z \leq-h_{1}-h_{2} .
\end{array}\right.
$$

Note that $\phi=C_{1}$ at the upper interface $z=-h_{1}$, and $\phi=C_{2}$ at the lower interface $z=-h_{1}-h_{2}$. The continuity of pressure across each interface is ensured by the jump conditions $c^{2}\left[\phi_{z}\right]_{(b)}^{(a)}+g^{\prime} \phi=0, g^{\prime}=g \Delta \rho / \rho_{2}$ :

$$
\begin{gathered}
c^{2}\left[C_{1}\left(\frac{1}{h_{1}}+\frac{1}{h_{2}}\right)-\frac{C_{2}}{h_{2}}\right]-g^{\prime} C_{1}=0, \\
c^{2}\left[C_{2}\left(\frac{1}{h_{2}}+\frac{1}{h_{3}}\right)-\frac{C_{1}}{h_{2}}\right]-g^{\prime} C_{2}=0, \\
2 g^{\prime} \lambda=\frac{2 g^{\prime}}{c^{2}}=\left(\frac{1}{h_{1}}+\frac{2}{h_{2}}+\frac{1}{h_{3}}\right) \mp\left[\left(\frac{1}{h_{1}}-\frac{1}{h_{3}}\right)^{2}+\frac{4}{h_{2}^{2}}\right]^{1 / 2} .
\end{gathered}
$$

The signs $\mp$ correspond to mode- 1 and mode- 2 respectively, so that, as required $c_{1}>c_{2}$. Note that $\lambda>0$ for both modes as required. It then follows that

$$
\frac{C_{1}}{C_{2}}=R=H \pm\left(H^{2}+1\right)^{1 / 2}, \quad \frac{C_{2}}{C_{1}}=\frac{1}{R}=-H \pm\left(H^{2}+1\right)^{1 / 2},
$$




$$
H=\frac{h_{2}}{2}\left(\frac{1}{h_{3}}-\frac{1}{h_{1}}\right) .
$$

The solution is normalised by $\max [|\phi|]=1$, so that $\max \left[\left|C_{1}\right|,\left|C_{2}\right|\right]=1$. For mode-1, $C_{1,2}>0$ and either the maximum of $|\phi(z)|$ occurs at the upper interface if $H>0, h_{1}>h_{3}$, $1=C_{1}>C_{2}>0$, or at the lower interface if $H<0, h_{1}<h_{3}, 1=C_{2}>C_{1}$. For mode-2, $C_{1} C_{2}<0$ and the maximum of $|\phi(z)|$ occurs at the lower interface if $H>0,1=C_{2}>0>$ $C_{1}>-1$, or at the upper interface if $H<0,1=C_{1}>0>C_{2}>-1$. Thus if a mode-2 wave is a wave of elevation, then this corresponds to convex wave if the maximum of $|\phi(z)|$ occurs at the upper interface, $H<0, h_{1}<h_{3}$, but corresponds to a concave wave if the maximum of $|\phi(z)|$ occurs at the lower interface, $H>0, h_{1}>h_{3}$. This description is reversed if it is a wave of depression, which then corresponds to a concave wave if the maximum of $|\phi(z)|$ occurs at the upper interface, $H<0, h_{1}<h_{3}$, but corresponds to convex wave if the maximum of $|\phi(z)|$ occurs at the lower interface, $H>0, h_{1}>h_{3}$.

It is useful to take the limit $h_{2} \rightarrow 0$ so that the three-layer system reduces to a two-layer sytem with layer depths $h_{1}, h_{3}$. In that limit $H \rightarrow 0$ and from (B.4) $R=C_{1} / C_{2} \rightarrow \pm 1$. For a mode- 1 wave $C_{1}=C_{2}=1$, and for mode- 2 wave $C_{1}= \pm 1, C_{2}=\mp 1$, depending on whether $h_{1}<h_{3}$ or $h_{3}>h_{1}$. The speeds are found from (B.3c) as $c^{2}=2 g^{\prime} h_{1} h_{3} / h$, for modes 1 and 2 respectively. In this limit as $h_{2} \rightarrow 0$, we obtain,

$$
I_{11}^{(a),(b)} \approx \frac{1}{h_{1}}+\frac{1}{h_{3}^{(b)}}, \quad I_{1}^{(a)} \approx \frac{1}{h_{1}}+\frac{1}{h_{3}^{(a)}}, \quad I_{1}^{(b)} \approx \frac{1}{h_{1}}+\frac{1}{h_{3}^{(a)}} .
$$

Hence, from (20) we recover the expressions (25).

The $\mathrm{KdV}$ coefficients $\mu, \delta$ in $(1)$ are given by $(3 a, b)$ and after subsituting for the modal finction from (B.2) we determine

$$
\begin{aligned}
& \mu=3 c^{2}\left[-\frac{C_{1}^{3}}{h_{1}^{2}}+\frac{C_{2}^{3}}{h_{3}^{2}}+\frac{\left(C_{1}-C_{2}\right)^{3}}{h_{2}^{2}}\right] / I, \\
& \delta=c^{2}\left[C_{1}^{2} \frac{h_{1}+h_{2}}{3}+C_{2}^{2} \frac{h_{3}+h_{2}}{3}+C_{1} C_{2} \frac{h_{2}}{3}\right] / I,
\end{aligned}
$$

where

$$
I=2 c\left[\frac{C_{1}^{2}}{h_{1}}+\frac{C_{2}^{2}}{h_{3}}+\frac{\left(C_{1}-C_{2}\right)^{2}}{h_{2}}\right] .
$$

It is well known that if $\mu_{1}>0(<0)$ then mode- 1 solitary waves are waves of elevation (depression). However, if $\mu_{2}>0(<0)$ implying that mode-2 solitary waves are waves of elevation (depression), then this may corresponds to a convex or a concave wave depending on the location of the maximum of $\left|\phi_{2}\right|$ as described above.

\section{Appendix C: Mode generation by a mode-2 incident wave}

Although the case of a mode- 2 wave incident on a step has less interest due to the rarity of observed mode- 2 waves compared to mode- 1 waves, the theory presented in section 3 can be used to describe this case. All that is required is to set $k=2, N=2$ in (23), and the outcome for a three-layer fluid is shown in tables C1, C2, C3 for the same parameter settings used for the case of a mode- 1 incident wave. Overall the results are similar to those for the case of a mode- 1 incident wave with the roles of mode- 1 and mode- 2 interchanged. That is, in the first two cases, the dominant feature is the transmitted mode- 2 wave, while in the third case 
Table C1. Case 1: Reflection and transmission coefficients for (14) for an incident mode-2 wave, and the energy fluxes (18) in units of $\mathrm{ms}^{-3}$.

\begin{tabular}{c|ccccccc}
\hline \hline Case 1 & $S_{1}$ & $R_{1}$ & $S_{2}$ & $R_{2}$ & $J^{(i n)}$ & $J^{(a)}$ & $J^{(b)}\left(* 10^{-5}\right)$ \\
\hline \hline$h_{2}=40 \mathrm{~m}$ & 0.0156 & -0.0072 & 0.9832 & 0.0044 & 0.0076 & 0.0076 & -0.1408 \\
$h_{2}=60 \mathrm{~m}$ & 0.0288 & -0.0112 & 0.9705 & 0.0068 & 0.0087 & 0.0087 & -0.3340 \\
$h_{2}=80 \mathrm{~m}$ & 0.0473 & -0.0159 & 0.9523 & 0.0096 & 0.0095 & 0.0095 & -0.6545 \\
\hline
\end{tabular}

Table C2. Case 2: Reflection and transmission coefficients for (14) for an incident mode-2 wave, and the energy fluxes (18) in units of $\mathrm{ms}^{-3}$.

\begin{tabular}{c|ccccccc}
\hline \hline Case 2 & $S_{1}$ & $R_{1}$ & $S_{2}$ & $R_{2}$ & $J^{(i n)}$ & $J^{(a)}$ & $J^{(b)}\left(* 10^{-4}\right)$ \\
\hline \hline$h_{1}=160 \mathrm{~m}$ & 0.0823 & -0.0246 & -1.0215 & 0.0166 & 0.0105 & 0.0105 & -0.1846 \\
$h_{1}=180 \mathrm{~m}$ & 0.1169 & -0.0311 & -1.0917 & 0.0220 & 0.0110 & 0.0109 & -0.3160 \\
$h_{1}=200 \mathrm{~m}$ & 0.1747 & -0.0403 & -1.1734 & 0.0302 & 0.0114 & 0.0113 & -0.5658 \\
\hline
\end{tabular}

Table C3. Case 3: Reflection and transmission coefficients for (14) for an incident mode-2 wave, and the energy fluxes (18) in units of $\mathrm{m} \mathrm{s}^{-3}$.

\begin{tabular}{c|ccccccc}
\hline \hline Case 3 & $S_{1}$ & $R_{1}$ & $S_{2}$ & $R_{2}$ & $J^{(i n)}$ & $J^{(a)}$ & $J^{(b)}\left(* 10^{-3}\right)$ \\
\hline \hline$h_{1}=220 \mathrm{~m}$ & -0.2737 & 0.0544 & 1.2494 & 0.0434 & 0.0114 & 0.0113 & -0.1058 \\
$h_{1}=240 \mathrm{~m}$ & -0.4476 & 0.0790 & 1.3085 & 0.0683 & 0.0110 & 0.0107 & -0.2209 \\
$h_{1}=260 \mathrm{~m}$ & -0.7220 & 0.1360 & 1.3505 & 0.1346 & 0.0105 & 0.0099 & -0.6689 \\
\hline
\end{tabular}

there is significant energy transfer to mode 1 . With the reflection and transmission coefficients determined, we could now describe the evolution away from the step as in section 4 , but the details are omitted here. As in section 4 the qualitative outcomes can be predicted using the knowledge of the reflected and transmitted wave amplitudes and the KdV coefficients. 